\title{
Pathogenesis of Beta-lactam-induced Serum Sickness-Like Reaction: The potential role of Reactive Drug Metabolites
}

\author{
Abdelbaset Elzagallaai $^{1}$, Awatif Abuzgaia ${ }^{1}$, Blanca Rosa Del Pozzo Magaña ${ }^{1}$, Eman \\ Loubani $^{1}$, and Michael Rieder ${ }^{2}$ \\ ${ }^{1}$ Western University \\ ${ }^{2}$ University of Western Ontario
}

April 28, 2020

\begin{abstract}
Aims: Drug-induced serum sickness-like reactions (SSLRs) are idiosyncratic drug-induced hypersensitivity reactions that occur in susceptible patients 1-3 weeks after exposure to the culprit drug. The pathophysiology of this type of reactions is not well understood and its diagnosis is difficult due to the lack of safe and reliable diagnostic tests for identifying the culprit drug. The lymphocyte toxicity assay (LTA) is an in vitro test used as a diagnostic and investigative tool for drug hypersensitivity reactions (DHRs). In this pilot study, we investigated the pathogenesis of SSLR using the LTA test to evaluate the potential role of reactive drug metabolites in the pathogenesis of SSLR. Methods: Nineteen patients (14 males and 5 females) were recruited to this study. Demographic data was collected form the patents and blood samples were withdrawn from all patients and from 19 healthy controls. The LTA test was performed on all subjects and data is expressed as percentage increase in cell death compared to control (vehicle without the drug). Results: There was a significant $(\mathrm{p}<0.05)$ concentration-related increase in cell death in cells isolated from patients as compared to cells from healthy controls when incubated with the drug in the presence of phenobarbitone-induced rat liver microsomes (MICs). Conclusion: This data suggests the initial bioactivation of the drug to a reactive metabolite followed by a toxic response is a key first step in ?-lactam antibiotic-induced SSLRs. Further research is needed to explore the implications of this data as to the pathogenesis of ?-lactam antibiotic induced SSLR.
\end{abstract}

\section{Introduction}

Serum sickness (SS) is a type III immune-complex mediated reaction first described by von Pirquet and Schick in 1951 when they recognized reactions developed in susceptible individuals 8-12 days after receiving heterologous antisera for the treatment of diphtheria and scarlet fever ${ }^{1}$. It was later found that circulating immune complexes and complement activation is important in the pathophysiology of these immune-mediated reactions $^{2,3}$. SS is characterized by fever, cutaneous eruptions, arthralgia and lymphadenopathy. Serum sickness-like reaction (SSLR) is clinically similar reaction associated with commonly used drugs including beta-lactam antibiotics (especially cefaclor), sulfonamides, fluoroquinolones, aromatic anticonvulsants, tetracyclines, metronidazole, bupropion and others ${ }^{2,4-6}$. It is defined by rash (usually urticaria) and arthritis usually manifested 1-3 weeks after drug exposure, which can be accompanied by lymphadenopathy, eosinophilia and rarely renal involvement ${ }^{7}$. It is uncommonly seen in clinical practice, but its incidence appears to be on the rise since the introduction of biologic drugs ${ }^{8-10}$. It has been estimated that the incidence of SSLR associated with cefaclor was between $0.024 \%$ and $0.2 \%$ per course $^{7}$.

The precise pathophysiology of drug-induced SSLR is not well understood. However, in delayed onset drug hypersensitivity the generation of cytotoxic reactive metabolites from drug molecules in vivo is believed to be the first step in a cascade of events leading to the immune-mediated reaction ${ }^{11}$. These reactive metabolites are capable of adducting (hapenating) endogenous macromolecules produced an antigen recognized by the 
immune system as non-self. They may also cause local or systemic cell damage resulting in releasing 'danger signals', which prime immune cells to mount the reaction ${ }^{12,13}$. Diagnosis of drug-induced SSLR is mainly based on clinical presentation and medication history and no reliable and safe diagnostic test is available to date. The lymphocyte toxicity assay (LTA) is an in vitro test that has been proven to have a significant value in the diagnosis of drug-induced hypersensitivity reactions (DHRs) but most of the validation work has been focused on type IV T-cell-mediated delayed DHRs. In this study we set to explore the potential role(s) of reactive drug metabolites in $\beta$-lactam antibiotic induced SSLR using a cohort of patients and healthy control volunteers.

\section{Materials:}

Penicillin, cefazolin, tetrazolium salt 3-(4, 5-dimethylthiazol-2-yl) 2, 5 diphenyl-tetrazolium bromide (MTT), hydrogen peroxide $\left(\mathrm{H}_{2} \mathrm{O}_{2}\right), 2$ ', 7 '-dichlorofluorescin diacetate (DCFH-DA), Histopaque@ -1077 (Ficoll), Hank's balanced salt solution (HBSS) and dimethyl sulfoxide (DMSO) were purchased from Sigma-Aldrich (St, Louis, MO, USA). RPMI 1640 and trypan blue were purchased from Invitrogen, Life Technologies Inc. (Burlington, ON, Canada). All other chemicals used in this study were the highest purity commercially available.

\section{Subjects:}

Informed consent was obtained from all participants and the study protocol was approved by the Western University Research Ethics Board for human subjects (REB No. 11883E) as well as the London Health Sciences Research Ethics Board. Three groups of individuals were included in our research study. The first group consisted of patients who had experienced a $\beta$-lactam antibiotic-induced SSLR. These individuals developed symptoms highly suggestive of SSLR as a result of $\beta$-lactam antibiotic administration. Overall, 19 $\beta$-lactam antibiotic hypersensitive (BLHS) patients between the age of 7 and 77 years were recruited. The patients' symptoms are summarized in Table 1. PMBCs isolated from each of these demonstrated dose-related toxicity in a lymphocyte toxicity assay (LTA) assessing susceptibility to reactive drug metabolites. The second study group consisted of 19 healthy volunteers who have denied any history of drug hypersensitivity reactions or exposure to beta-lactams.

\section{Blood Collection and Isolation of Cells:}

Peripheral venous blood samples were collected from each participant by venipuncture into heparinized syringes and processed immediately. To isolate peripheral blood monocytes (PBMCs), blood was diluted 1:1 with phosphate-buffered saline (PBS, $10 \mathrm{mM} \mathrm{NaH}_{2} \mathrm{PO}_{4}, 2 \mathrm{mM} \mathrm{KH_{2 }} \mathrm{PO}_{4}, 137 \mathrm{mM} \mathrm{NaCl}, 2.7 \mathrm{mM} \mathrm{KCl} ; \mathrm{pH}$ 7.2 ) and $30 \mathrm{~mL}$ were layered over $15 \mathrm{~mL}$ of Ficoll-Paque density gradient and centrifuged at $500 \mathrm{~g}$ for 20 min. The interface layer (buffy coat) was then collected. Cells were washed twice with PBS and adjusted to $1 \times 10^{6} \mathrm{cell} / \mathrm{mL}$ in HEPES (4-(2-hydroxyethyl)-1-piperazine) ethanesulfonic acid buffered saline containing $15 \mathrm{mM}$ HEPES, $125 \mathrm{mM} \mathrm{NaCl}, 6 \mathrm{mM} \mathrm{KCl}, 1.2 \mathrm{mM} \mathrm{MgSO}_{4}, 1.0 \mathrm{mM} \mathrm{NaHCO}_{3}, 1.0 \mathrm{mM} \mathrm{CaCl} 2,10 \mathrm{mM}$ glucose; $\mathrm{pH} 7.4)$.

\section{In vitro toxicity testing}

The LTA was performed as described previously. ${ }^{14,15}$ Briefly, PBMCs were plated in flat-bottom 96-multi-well plates at a density of $1 \times 10^{5}$ cells per well in quadruplicate and treated with a final concentration of $800 \mu \mathrm{M}$ SMX or increasing concentrations of SMX-HA of $25,50,100,200,400$, or $800 \mu \mathrm{M}$ for $2 \mathrm{hr}$ at $37^{\circ} \mathrm{C}$ in a $5 \% \mathrm{CO}_{2}$ humidified atmosphere. SMX and SMX-HA solutions were freshly prepared in dimethyl sulfoxide (DMSO) and diluted in culture media to give the desired final concentration of drug or RM (DMSO final concentration is always kept at [?]1\%). A standard curve for measuring cell death was generated by seeding cells at $25 \%, 50 \%, 75 \%$ or $100 \%$ of cell concentration in culture media in quadruplicate. After incubation, drugs in solution were removed by centrifugation at $500 \mathrm{x}$ g for $10 \mathrm{~min}$. Then, cells were suspended in 100 $\mu \mathrm{L}$ fresh RPMI-1640 media supplemented with $10 \% \mathrm{FBS}, 100 \mathrm{U} / \mathrm{mL}$ penicillin $\mathrm{G}$ sodium and $100 \mu \mathrm{g} / \mathrm{mL}$ streptomycin sulfate, and left to recover for 18 hours in an atmosphere of $5 \% \mathrm{CO}_{2}$ at $37^{\circ} \mathrm{C}$. Cell viability was quantified using MTT staining as described previously. ${ }^{14}$

The iPTA was performed in similar fashion as the LTA (above) except that calcium free Locke's solution was 
used as the medium. Platelets were isolated from blood samples as described above and plated at a density of $1 \times 10^{7}$ cells/well. To pellet platelets after treatment plates were centrifuged at $900 \times \mathrm{g}$ for $15 \mathrm{~min}$.

\section{Statistical Analysis}

Statistical analysis was performed using SPSS. The numbers of dead cells were expressed as a percentage of control (vehicle without drug) and blotted as mean \pm standard error (SEM). Significant differences were determined by Student's t-test with Bonferroni correction for multiple comparisons. A probability of more than $95 \%$ (P[?]0.05) was considered significant. Correlations were made using Pearson correlation analyses. Unless otherwise indicated, values are presented as mean +- standard error (SEM).

\section{Results}

Nineteen patients (14 males and 5 females) presented with symptoms highly suspicious for SSLR to betalactam antibiotics including penicillins and cephalosporins. Clinical symptoms included joint inflammation (arthritis) that included hands, feet, or both and cutaneous lesions (maculopapular, EM, urticaria). The mean age of the patients was 9.9 years and ranged from 11 months to 67 years. The characteristics of the patient population is summarized in Table 1. When blood samples were obtained from the 19 patients and 19 other age and gender-matched healthy volunteer cohort, all patients had positive LTA test results using a cut-off value of $20 \%$ increase in cell death (Figure 1). At $125 \mu \mathrm{M}$ of the drug and in the presence of phenobarbitone-induced rat liver microsomes $(\mathrm{MICs})$, degree of cell death was significantly higher $(\mathrm{p}<0.05)$ in cells isolated from beta-lactam-induced SSLR patients $(48 \% \pm 5.6)$ than cells from healthy controls $(8 \% \pm 4.6)$.

\section{Discussion}

Drug-induced SSLR represent a major problem - along with other idiosyncratic hypersensitivity reactions to management of infectious diseases due to the difficulty in timely confirmation the diagnosis and accurate identification of the culprit drug. Approximately $10 \%$ of the general population report an allergy to $\beta$-lactam antibiotics; however, $90 \%$ of reported allergies to $\beta$-lactam antibiotic cannot be confirmed immunologically 16. Such false labeling of patients puts them at greater risk of adverse reaction due to the use of less safe alternatives to treat their infection which increases length of hospital stay and likelihood of bad outcomes. Furthermore, resorting to non-beta-lactam antibiotics increases the cost burden of healthcare and contributes to worsening the bacterial resistance problem.

The pathophysiology of SSLR to $\beta$-lactam antibiotics is unclear (Figure 2). However, many cases of druginduced delayed hypersensitivity appear to evolve due to increased toxicity of reactive drug metabolites followed by a mis-directed immune response. We have demonstrated that there is a difference in cellular capacity with respect to defense with reactive drug metabolites between patients who have sustained a SSLR to $\beta$-lactam antibiotics versus the cells of healthy volunteers. This implies that dealing with reactive drug metabolite burden may be a critical first step in the pathogenesis of SSLR to $\beta$-lactam antibiotics. We have previously demonstrated this with respect to cefaclor and these findings suggest that this may be a common element in the pathogenesis of serious delayed adverse drug reactions to $\beta$-lactam antibiotics, supporting the importance of initial bioactivation of the drug leading to a misdirected immune response resulting in delayed drug hypersensitivity manifested as a SSLR ${ }^{5}$.

In addition to informing pathogenesis this has implications for diagnostic assessments. All the available diagnostic aids including skin testing and oral re-challenge have their risks and shortcomings and are not always feasible to perform either due to lack of expertise or fear of inducing a severe reaction in the patient. The LTA has the advantage of being safe as an in vitro test and can be used both as a diagnostic test and an investigative tool for the pathophysiology of SSLRs. Kearns et al. ${ }^{5}$ tested 19 patients (10 male and 9 females) suspected of developing SSLR to cefaclor and found that subjects with SSLR exhibited an increase in cell death of $50 \%$ to $167 \%$ above baseline. The effect was specific to cefaclor and was not produced by incubation of isolated cells with another cephalosporin (cephalexin) along with metabolic activation system 5. In another study, the same group also tested 10 patients with SSLR to cefaclor using the LTA test. The degree of cell death in the patient pollution was highly positive and ranged from $40 \%$ to $140 \%$ increase above 
baseline ${ }^{17}$. In a validation study for the LTA test using systemic re-exposure as a gold standard to determine the predictive value of the test for diagnosis of hypersensitivity reactions (HSRs) to different groups of drugs, we tested 11 patients with HSRs to beta-lactam antibiotics (6 to amoxicillin and 5 to cefaclor) ${ }^{14}$. When the results of the re-exposure were compared to the LTA results, all except one patient had complete agreement.

Data presented here points to the value of the LTA to explore mechanism(s) of drug hypersensitivity and potentially as a diagnostic tool for beta-lactam-induced SSLRs. Further research with larger numbers of patients is needed to further explore the pathophysiology and biology of SSLR to to $\beta$-lactam antibiotics.

\section{Data availability statement:}

Data is available upon request.

\section{Acknowledgements:}

Author contribution' statement: AAE designed the study, analyze the data and wrote the manuscript. AMA Collect data and blood samples and performed the in vitro testing, BDM recruit patients and collected clinical data. EM recruit patients and healthy controls to the study and revised the manuscript. MJR contributed to the original design of the study, recruitment of patients and writing of the manuscript.

This research was funded by the GSK-CIHR Chair in Paediatric Clinical Pharmacology to MJR.

\section{References}

1. von Pirqet C, Schick BT. Serum Sickness. Baltimore, MD: Williams \& Wilkins Co; 1951.

2. Lawley TJ, Bielory L, Gascon P, Yancey KB, Young NS, Frank MM. A prospective clinical and immunologic analysis of patients with serum sickness. N Engl J Med. 1984;311(22):1407-1413.

3. Vaughan JH, Barnett EV, Leadley PJ. Serum sickness. Evidence in man of antigen-antibody complexes and free light chains in the circulation during the acute reaction. Ann Intern Med. 1967;67(3):596-602.

4. Heckbert SR, Stryker WS, Coltin KL, Manson JE, Platt R. Serum sickness in children after antibiotic exposure: estimates of occurrence and morbidity in a health maintenance organization population. Am $J$ Epidemiol. 1990;132(2):336-342.

5. Kearns GL, Wheeler JG, Childress SH, Letzig LG. Serum sickness-like reactions to cefaclor: role of hepatic metabolism and individual susceptibility. J Pediatr. 1994;125(5 Pt 1):805-811.

6. Platt R, Dreis MW, Kennedy DL, Kuritsky JN. Serum sickness-like reactions to amoxicillin, cefaclor, cephalexin, and trimethoprim-sulfamethoxazole. J Infect Dis. 1988;158(2):474-477.

7. Knowles SR, Uetrecht J, Shear NH. Idiosyncratic drug reactions: the reactive metabolite syndromes. Lancet. 2000;356(9241):1587-1591.

8. Khan DA. Hypersensitivity and immunologic reactions to biologics: opportunities for the allergist. Ann Allergy Asthma Immunol.2016;117(2):115-120.

9. Yorulmaz A, Akin F, Sert A, Agir MA, Yilmaz R, Arslan S. Demographic and clinical characteristics of patients with serum sickness-like reaction. Clin Rheumatol. 2018;37(5):1389-1394.

10. Finger E, Scheinberg M. Development of serum sickness-like symptoms after rituximab infusion in two patients with severe hypergammaglobulinemia. J Clin Rheumatol. 2007;13(2):94-95.

11. Elzagallaai AA, Greff M, Rieder MJ. Adverse Drug Reactions in Children: The Double-Edged Sword of Therapeutics. Clin Pharmacol Ther. 2017;101(6):725-735.

12. Matzinger P. Tolerance, danger, and the extended family. Annu Rev Immunol. 1994;12:991-1045.

13. Pichler WJ, Adam J, Daubner B, Gentinetta T, Keller M, Yerly D. Drug hypersensitivity reactions: pathomechanism and clinical symptoms.Med Clin North Am. 2010;94(4):645-664, xv. 
14. Elzagallaai AA, Jahedmotlagh Z, Del Pozzo-Magana BR, et al. Predictive value of the lymphocyte toxicity assay in the diagnosis of drug hypersensitivity syndrome. Mol Diagn Ther.2010;14(5):317-322.

15. Elzagallaai AA, Rieder MJ, Koren G. The in vitro platelet toxicity assay (iPTA): a novel approach for assessment of drug hypersensitivity syndrome. J Clin Pharmacol. 2011;51(3):428-435.

16. Surtees SJ, Stockton MG, Gietzen TW. Allergy to penicillin: fable or fact? Bmj. 1991;302(6784):10511052 .

17. Kearns GL, Wheeler JG, Rieder MJ, Reid J. Serum sickness-like reaction to cefaclor: lack of in vitro cross-reactivity with loracarbef. Clin Pharmacol Ther. 1998;63(6):686-693.

Legends to figures:

\section{Legend to Figure 1}

Figure (1): Summary of the LTA test results for 19 SSLR patients and 19 healthy controls. Lymphocytes isolated from health controls were incubated with drug either without microsomes (MICs, white bars) or with drug and MICs (light grey bars) and cells isolated from SSLR patients were incubated with drug either without MICs (dark grey bars) or with MICs (black bars). Y axis represents cell viability expressed as a percentage from incubating the corresponding cells with control vehicle without the drug. ${ }^{*}, \mathrm{p}<0.05$; **, $\mathrm{p}<0.01$.

\section{Legend to Figure 2}

Figure (2): Proposed mechanisms of delayed drug hypersensitivity. This data supports the role of reactive metabolites in the pathogenesis of SSLR to $\beta$-lactam antibiotics and the key role of the hapten hypothesis starting with bioactivation of the drug to a reactive metabolite and following immune processing resulting in a mis-directed immune response producing delayed drug hypersensitivity manifested as a SSLR.

\section{Hosted file}

Table 1.docx available at https://authorea.com/users/309261/articles/440212-pathogenesis-ofbeta-lactam-induced-serum-sickness-like-reaction-the-potential-role-of-reactive-drug-metabolites

\section{Hosted file}

Figure 1.docx available at https://authorea.com/users/309261/articles/440212-pathogenesis-ofbeta-lactam-induced-serum-sickness-like-reaction-the-potential-role-of-reactive-drug-metabolites

\section{Hosted file}

Figure 2.docx available at https://authorea.com/users/309261/articles/440212-pathogenesis-ofbeta-lactam-induced-serum-sickness-like-reaction-the-potential-role-of-reactive-drug-metabolites 